\title{
Invited commentary: advantages and cautions for developing valid prognostic models of simultaneous pancreas and kidney transplantation outcomes
}

\author{
Arianeb Mehrabi ${ }^{1,2} \cdot$ Elias Khajeh $^{1,3} \cdot$ Martin Zeier $^{4}$
}

Received: 2 November 2018 / Accepted: 5 November 2018 / Published online: 12 November 2018

(C) Springer-Verlag GmbH Germany, part of Springer Nature 2018

By invitation from the Editor in Chief, we wish to comment on the article, "Development and validation of a prognostic model for kidney function one year after combined pancreas and kidney transplantation using pre-transplant donor and recipient variables," by Zorn et al. [1]. In their article, Zorn et al. presented a prognostic model for the prediction of kidney function 1 year after simultaneous pancreas and kidney transplantation (SPKT) using pre-transplant donor and recipient variables. SPKT improves the survival and provides a significantly better quality of life for patients with diabetes by removing the need for dialysis and insulin injections $[2,3]$. The need for SPKT will increase in the future because the incidence of diabetes is continuously increasing. However, despite remarkable efforts to overcome the organ shortage in recent decades, the number of available organs still falls short of the demand. Moreover, prognostic models to avoid suboptimal donor and recipient matches are lacking. All these points make the present paper from Zorn et al. (which deals with transplantation equity in organ allocation in the face of organ shortages) valid and interesting.

In their study, the authors demonstrated that not only preoperative recipient variables but also donor variables are

Arianeb Mehrabi

arianeb_mehrabi@med.uni-heidelberg.de

1 Department of General, Visceral, and Transplantation Surgery, University of Heidelberg, Heidelberg, Germany

2 Division of Visceral Transplantation, Department of General, Visceral, and Transplantation Surgery, University of Heidelberg, Im Neuenheimer Feld 110, 69120 Heidelberg, Germany

3 Medical Statistics Group, Department of General, Visceral, and Transplantation Surgery, University of Heidelberg, Heidelberg, Germany

4 Division of Pancreas and Kidney Transplantation, Department of Nephrology, Heidelberg University Hospital, Heidelberg, Germany relevant for the prediction of kidney graft function after 1 year. They showed that older male recipients had a higher risk of poor kidney graft function 1 year after SPKT. However, a male donor organ was an independently protective factor of kidney graft function 1 year after SPKT. Older donor age, higher donor serum urea levels, and higher donor glomerular filtration rate values increased the risk of poor kidney graft function. The authors suggested avoiding combining these donor-related risk factors with high-risk recipients.

With respect to the great work of Zorn et al., there are some important issues which should be kept in mind when interpreting the results of their study. The first critical issue is that the present model predicts only renal function after SPKT. Although renal function is important in diabetic recipients, kidney and pancreas function and outcome should both be assessed after SPKT. Pancreas graft failure, especially in the early post-transplantation period, and pancreas-related complications often result in reoperation $[4,5]$ and hence have a major impact on kidney graft function after SPKT [6-8]. Therefore, a prognostic model for SPKT that can predict the probability of both pancreas and kidney failure would be more useful when making decisions about graft allocation.

The major limitation of the present study is the high rate of excluded patients due to loss to follow-up or lack of data. It is not clear from the flowchart of the training cohort how many patients receiving SPKT were excluded. However, it is shown that more than 25\% (468 out of 1785) of patients receiving a kidney transplant from a deceased donor were excluded from the study due to lack of sufficient follow-up. It has been suggested that a loss of more than $20 \%$ of patients poses a serious threat to the validity of the study findings. The high dropout rate is a major source of bias in this study because dropout rates are usually different between study groups, and the patients who drop out are different from those who complete the follow-up [9]. 
Lack of information on the cause of death in $49.5 \%$ of donors is an additional limitation of the study. Cause of donor death plays a major role in graft quality and outcomes after kidney transplantation. The cause of donor death is associated with renal function of the donor because adequate renal function protects against adverse cardiovascular events [10]. The authors have mentioned this as a limitation of their study, and it reveals the suboptimal quality of data registration in EuroTransplant. With advances in information technology, data transfer, and data registry facilities, it is time to provide scientific, clinically based, standard, and easy to use datasheets to improve data acquisition in EuroTransplant centers. The data should be collected comprehensively using classifications that are clinically and scientifically defined. Another strong limitation of the study is the small sample size of 111 patients, including 81 recipients with KDIGO (Kidney Disease: Improving Global Outcomes) $\geq$ III versus 30 recipients with KDIGO < III as an outcome. Logistic regression analysis tends to overestimate odds ratios in studies with small-to-moderate samples sizes [11]. There is a risk of overestimating the effect measure; therefore, the results should be interpreted cautiously.

Immune suppression can strongly affect kidney function after kidney transplantation; therefore, this should be taken into account in an inclusive model aiming to predict the outcomes after SPKT. Importantly, calcineurin inhibitors present different risks of developing kidney injury [12]. Tacrolimus has lower nephrotoxicity than cyclosporine and tacrolimus-based regimens and is associated with lower graft rejection and higher graft survival rates [13, 14]. Although the aim of this paper was to develop a model based on pre-transplant variables, the presented model should be adjusted for the effect of immunosuppression. At the very least, the model needs to be validated in a homogenous subgroup of patients receiving the same immunosuppressive regimen (preferentially the current standard regimen).

In summary, developing valid prognostic models of SPKT outcomes to avoid suboptimal matches of donors and recipients is valuable and important because there is a shortage of donor organs. The interesting model for predicting kidney function 1 year after SPKT presented by Zorn et al. should be interpreted cautiously because important data is lacking, such as the cause of donor death and details of the immunosuppressive regimen. In addition, the model suffers from a risk of bias because the sample size is small and the dropout rate is high. We suggest that the model be further validated in large and homogenous cohorts. This would improve the model and extend it to a prognostic model for SPKT based on both pancreas and kidney graft outcomes.
Acknowledgments We thank the reviewers of the article of Zorn et al., for their valuable comments, which we used for this invited commentary.

\section{Compliance with ethical standards}

Conflict of interest The authors declare that they have no conflicts of interest.

Ethical approval Not applicable.

Informed consent Not applicable.

\section{References}

1. Zorn KS, Littbarski S, Schwager Y, Kaltenborn A, Beneke J, Gwiasda J, Becker T, Braun F, Reichert B, Klempnauer J, Schrem $H$ (2018) Development and validation of a prognostic model for kidney function 1 year after combined pancreas and kidney transplantation using pre-transplant donor and recipient variables. Langenbeck's Arch Surg. https://doi.org/10.1007/s00423-0181712-z

2. Waki K, Terasaki PI, Kadowaki T (2010) Long-term pancreas allograft survival in simultaneous pancreas-kidney transplantation by era: UNOS registry analysis. Diabetes Care 33(8):1789-1791. https://doi.org/10.2337/dc09-2276

3. Morath C, Schmied B, Mehrabi A, Weitz J, Schmidt J, Werner J, Buchler MW, Morcos M, Nawroth PP, Schwenger V, Doehler B, Opelz G, Zeier M (2009) Simultaneous pancreas-kidney transplantation in type 1 diabetes. Clin Transpl 23(Suppl 21):115-120. https://doi.org/10.1111/j.1399-0012.2009.01116.x

4. Sollinger HW, Odorico JS, Becker YT, D'Alessandro AM, Pirsch JD (2009) One thousand simultaneous pancreas-kidney transplants at a single center with 22-year follow-up. Ann Surg 250(4):618630. https://doi.org/10.1097/SLA.0b013e3181b76d2b

5. Pieroni E, Napoli N, Lombardo C, Marchetti P, Occhipinti M, Cappelli C, Caramella D, Consani G, Amorese G, De Maria M, Vistoli F, Boggi U (2018) Duodenal graft complications requiring duodenectomy after pancreas and pancreas-kidney transplantation. Am J Transplant 18(6):1388-1396. https://doi.org/10.1111/ajt. 14613

6. Kaplan B, West-Thielke P, Herren H, Gill J, Knoll GA, Oberholzer J, Sankary H, Benedetti E (2008) Reported isolated pancreas rejection is associated with poor kidney outcomes in recipients of a simultaneous pancreas kidney transplant. Transplantation 86(9): 1229-1233. https://doi.org/10.1097/TP.0b013e318188ad11

7. Barlow AD, Saeb-Parsy K, Watson CJE (2017) An analysis of the survival outcomes of simultaneous pancreas and kidney transplantation compared to live donor kidney transplantation in patients with type 1 diabetes: a UK Transplant Registry study. Transpl Int 30(9):884-892. https://doi.org/10.1111/tri.12957

8. Morath C, Zeier M, Dohler B, Schmidt J, Nawroth PP, Opelz G (2008) Metabolic control improves long-term renal allograft and patient survival in type 1 diabetes. J Am Soc Nephrol: JASN 19(8):1557-1563. https://doi.org/10.1681/asn.2007070804

9. Dettori JR (2011) Loss to follow-up. Evid Based Spine Care J 2(1): 7-10. https://doi.org/10.1055/s-0030-1267080

10. Garcia-Gil M, Parramon D, Comas-Cufi M, Marti R, Ponjoan A, Alves-Cabratosa L, Blanch J, Petersen I, Elosua R, Grau M, Salvador B, Ramos R (2016) Role of renal function in cardiovascular risk assessment: a retrospective cohort study in a population with low incidence of coronary heart disease. Prev Med 89:200 206. https://doi.org/10.1016/j.ypmed.2016.06.004 
11. Nemes S, Jonasson JM, Genell A, Steineck G (2009) Bias in odds ratios by logistic regression modelling and sample size. BMC Med Res Methodol 9:56. https://doi.org/10.1186/1471-2288-9-56

12. Schmied BM, Muller SA, Mehrabi A, Welsch T, Buchler MW, Zeier M, Schmidt J (2006) Immunosuppressive standards in simultaneous kidney-pancreas transplantation. Clin Transpl 20(Suppl 17):44-50. https://doi.org/10.1111/j.1399-0012.2006.00599.x

13. Nankivell BJ, P'Ng CH, O'Connell PJ, Chapman JR (2016) Calcineurin inhibitor nephrotoxicity through the lens of longitudinal histology: comparison of cyclosporine and tacrolimus eras. Transplantation 100(8):1723-1731. https://doi.org/10.1097/ tp.0000000000001243

14. Boggi U, Marchetti P, Mosca F (2005) Is tacrolimus associated with fewer surgical complications than ciclosporin after kidney-pancreas transplantation? Nat Clin Pract Nephrol 1(2):76-77. https://doi.org/ 10.1038/ncpneph0043 